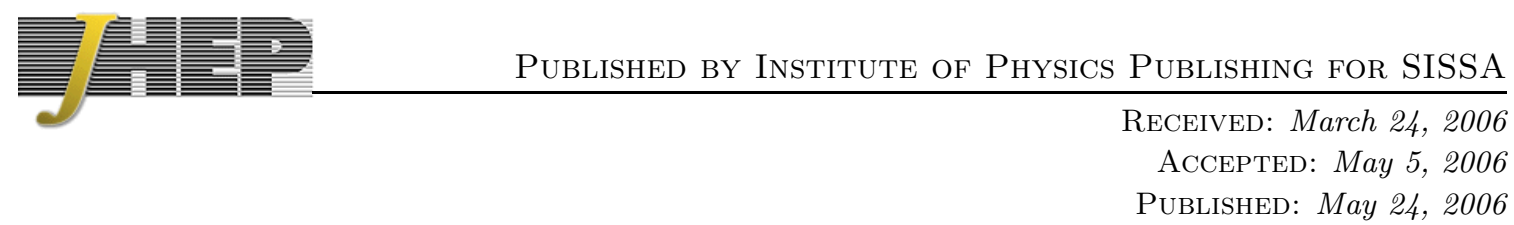

\title{
On $B_{s} \rightarrow \mu^{+} \mu^{-}$and cold dark matter scattering in the MSSM with non-universal Higgs masses
}

\author{
John Ellis, ${ }^{a}$ Keith A. Olive, ${ }^{b}$ Yudi Santoso $^{c}$ and Vassilis C. Spanos ${ }^{b}$ \\ ${ }^{a}$ TH Division, CERN \\ Geneva, Switzerland \\ ${ }^{b}$ William I. Fine Theoretical Physics Institute, University of Minnesota \\ Minneapolis, MN 55455, U.S.A. \\ ${ }^{c}$ Department of Physics and Astronomy, University of Victoria \\ Victoria, BC, V8P 1A1, Canada \\ E-mail: john.ellis@cern.ch, blive@physics.umn.edy, santoso@uvic.ca, \\ spanos@physics.umn.edu
}

ABStract: We show that present experimental constraints on $B_{s} \rightarrow \mu^{+} \mu^{-}$decay and the CDMS upper limit on the cold dark matter elastic scattering cross section already have significant impact on the parameter space of the minimal supersymmetric extension of the Standard Model (MSSM) with non-universal supersymmetry-breaking scalar masses for the Higgs multiplets (NUHM). The relaxation of scalar universality in the MSSM allows the possibility of a relatively light mass $M_{A}$ for the pseudoscalar Higgs boson. The present upper limit on $B_{s} \rightarrow \mu^{+} \mu^{-}$already excludes much of the scope for this possibility in the NUHM, in contrast to the constrained MSSM with universal scalar masses (CMSSM), where $B_{s} \rightarrow \mu^{+} \mu^{-}$decay does not exclude any ranges of parameters not already excluded by $b \rightarrow s \gamma$ decay. Cold dark matter scattering is also enhanced for small $M_{A}$, but the impact of present upper limit on $B_{s} \rightarrow \mu^{+} \mu^{-}$on the NUHM parameter space is in many cases greater than that of the CDMS scattering limit, particularly at large $\tan \beta$.

KEYwords: Supergravity Models, Supersymmetry Phenomenology. 


\section{Contents}

1. Introduction 1

2. Review of the calculations of $B_{s} \rightarrow \mu^{+} \mu^{-}$decay and spin-independent elastic $\chi$ scattering

3. Analysis of NUHM parameter planes 国

4. Summary and prospects 10

\section{Introduction}

Many phenomenological analyses of the parameter space of the MSSM assume universality for the soft supersymmetry-breaking scalar and gaugino masses, a theoretical framework often termed the constrained MSSM (CMSSM). However, this universality assumption is not necessarily supported by the effective supergravity models derived, for example, from string theory. On the other hand, the phenomenological suppression of flavour-changing neutral interactions suggests that squarks and sleptons with the same internal quantum numbers must be very nearly degenerate, at least for the supersymmetric partners of the first two generations, and there would be degeneracy (before renormalization) between squarks and sleptons in common GUT multiplets. However, there is no strong reason to suppose that the soft supersymmetry-breaking scalar masses $m_{i}^{2}, i=1,2$ of the Higgs multiplets should necessarily be the same as each other or the squarks and sleptons: $m_{i}^{2}=$ $\left(1+\delta_{i}\right) m_{0}^{2}$ with $\delta_{1,2} \neq 0$. These considerations motivate the phenomenological study of models with non-universal Higgs masses (NUHM) [1-6], as considered in this paper.

The parameter space of the NUHM has two dimensions more than the CMSSM that are spanned by $\delta_{1,2}$, allowing the Higgs supermultiplet mixing parameter $\mu$ and the pseudoscalar Higgs mass $M_{A}$ to be treated as parameters that are free, apart from theoretical constraints such as vacuum stability up to the scale of grand unification: to this end, we impose the requirement that $m_{i}^{2}+\mu^{2}>0$ at all renormalization scales below this GUT scale [4]. The phenomenological constraints on the NUHM provided by LEP constraints on the masses of the lightest supersymmetric Higgs boson $m_{h}$ []] and the lighter chargino $\chi^{ \pm}$have been considered, along with $b \rightarrow s \gamma$ decay [8, 9], the relic dark matter density $\Omega_{C D M} h^{2} \sqrt{10}$ and (optionally) the anomalous magnetic moment of the muon, $g_{\mu}-2$ [11]. These constraints allow regions of NUHM parameter space in which $M_{A}$ is considerably smaller than its value in the CMSSM.

The decay $B_{s} \rightarrow \mu^{+} \mu^{-}$is known to impose another interesting constraint on the parameter spaces of models for physics beyond the Standard Model, such as the MSSM [12[15]. The Fermilab Tevatron collider already has an interesting upper limit $\sim 2 \times 10^{-7}$ on 
the $B_{s} \rightarrow \mu^{+} \mu^{-}$decay branching ratio [16], and future runs of the Fermilab Tevatron collider and the LHC are expected to increase significantly the experimental sensitivity to $B_{s} \rightarrow \mu^{+} \mu^{-}$decay. However, a recent exploration of the present $B_{s} \rightarrow \mu^{+} \mu^{-}$constraint in the CMSSM 17 found that its impact was limited by uncertainties in the theoretical relation of $M_{A}$ to the underlying CMSSM parameters, and provided no extensions of the $\left(m_{1 / 2}, m_{0}\right)$ regions already excluded by $b \rightarrow s \gamma$ decay, in particular.

We explore in this paper the current impact of this additional constraint on the NUHM, and consider also the potential impact of future improvements in the experimental sensitivity to $B_{s} \rightarrow \mu^{+} \mu^{-}$decay within the NUHM. The rate for $B_{s} \rightarrow \mu^{+} \mu^{-}$may be enhanced in portions of the NUHM parameter space where $M_{A}$ is smaller than in the CMSSM. We find that, consequently, significant regions of the NUHM parameter space at small $M_{A}$ and large $\tan \beta$ are already excluded by the present experimental upper limit on $B_{s} \rightarrow \mu^{+} \mu^{-}$ decay. Likely improvements in sensitivity at the Fermilab Tevatron collider and the LHC will reach significant extra swathes of the NUHM parameter space.

The elastic cold dark matter scattering cross section is also enhanced at small $M_{A}$, and another important constraint on the NUHM parameter space is placed by the upper limit on the spin-independent cold dark matter scattering cross section from the CDMS Collaboration [18]. This has only just begun to cut into the CMSSM parameter space [19], but does impact the NUHM parameter space, as also discussed in this paper. However, in many of the specific cases studied, the $B_{s} \rightarrow \mu^{+} \mu^{-}$constraint is stronger than the CDMS constraint.

The structure of this paper is as follows. In Section 2 we recall briefly essential aspects of the theoretical calculation of $B_{s} \rightarrow \mu^{+} \mu^{-}$decay and spin-independent dark matter scattering. Then, in Section 3 we explore various slices through the NUHM parameter space, displaying the impact of the present experimental upper limit on $B_{s} \rightarrow \mu^{+} \mu^{-}$ decay. Finally, in Section 4 we discuss the potential impact of future improvements in the experimental sensitivity to this decay.

\section{Review of the calculations of $B_{s} \rightarrow \mu^{+} \mu^{-}$decay and spin-independent elastic $\chi$ scattering}

The branching ratio for the decay $B_{s} \rightarrow \mu^{+} \mu^{-}$is given by

$$
\begin{aligned}
\mathcal{B}\left(B_{s} \rightarrow \mu^{+} \mu^{-}\right) & =\frac{G_{F}^{2} \alpha^{2}}{16 \pi^{3}} \frac{M_{B_{s}}^{5} f_{B_{s}}^{2} \tau_{B}}{4}\left|V_{t b} V_{t s}^{*}\right|^{2} \sqrt{1-\frac{4 m_{\mu}^{2}}{M_{B_{s}}^{2}}} \\
& \times\left\{\left(1-\frac{4 m_{\mu}^{2}}{M_{B_{s}}^{2}}\right)\left|C_{S}\right|^{2}+\left|C_{P}-2 C_{A} \frac{m_{\mu}}{M_{B_{s}}^{2}}\right|^{2}\right\},
\end{aligned}
$$

where the one-loop corrected Wilson coefficients $C_{S, P}$ are taken from [20] and $C_{A}$ is defined in terms of $Y\left(x_{t}\right)$, following [21], as $C_{A}=Y\left(x_{t}\right) / \sin ^{2} \theta_{W}$ where

$$
Y\left(x_{t}\right)=0.99\left(\frac{m_{t}\left(m_{t}\right)}{165 \mathrm{GeV}}\right)^{1.55} .
$$


The function $Y\left(x_{t}\right)$ incorporates both leading [22] and next-to-leading order [23] QCD corrections, and $m_{t}\left(m_{t}\right)$ is the running top-quark mass in the $\overline{M S}$ scheme. We assume here that the physical top quark mass is $m_{t}=172.7 \pm 2.9 \mathrm{GeV}$ [24].

The Wilson coefficients $C_{S, P}$ receive four contributions in the context of MSSM, due to Higgs doublets [21], counter-terms, box and penguin diagram [25, 26] ${ }^{1}$. We have considered all these one-loop corrections as well as the dominant NLO QCD corrections studied in [27]. In addition, we have included the flavour-changing gluino contribution [28, 29]. The Wilson coefficients $C_{S, P}$ have been multiplied by $1 /\left(1+\epsilon_{b}\right)^{2}$, where $\epsilon_{b}$ incorporates the full flavour-independent supersymmetric one-loop corrections to the bottom-quark Yukawa coupling [30-32], that in principle are significant in the large-tan $\beta$ regime [12, 13]. Furthermore, it is known that the flavour-violating contributions arising from the Higgs and chargino couplings at the one-loop level result in effective one-loop corrected values for the Kobayashi-Maskawa (KM) matrix elements [26, 33]. These corrections modify the Wilson coefficients involved in Eq. (2.1), as can be seen in Eqs. (6.35) and (6.36) in 34 or in Eq. (14) in [28]. We have included these flavour-violating effects as described in [34, 28], taking into account the squark mixing effects.

The counter-terms are mediated by $A, H, h$ exchange, as seen in Eqs. (5.1) and (5.2) of [20], and dominate in the large-tan $\beta$ limit, where the $B_{s} \rightarrow \mu^{+} \mu^{-}$decay amplitude $\propto 1 / m_{A}^{2}$ and hence the decay rate $\propto 1 / m_{A}^{4}$. This underlines the potential of $B_{s} \rightarrow \mu^{+} \mu^{-}$ decay for constraining models with large $\tan \beta$ and small $m_{A}$.

As already noted, in the NUHM $M_{A}$ may take values different from those required by the vacuum conditions in the CMSSM. Quite generally, the electroweak vacuum conditions in the MSSM may be written in the form

$$
\mu^{2}=\frac{m_{1}^{2}-m_{2}^{2} \tan ^{2} \beta+\frac{1}{2} m_{Z}^{2}\left(1-\tan ^{2} \beta\right)+\Delta_{\mu}^{(1)}}{\tan ^{2} \beta-1+\Delta_{\mu}^{(2)}},
$$

and

$$
M_{A}^{2}(Q)=m_{1}^{2}(Q)+m_{2}^{2}(Q)+2 \mu^{2}(Q)+\Delta_{A}(Q),
$$

and $m_{1,2} \equiv m_{1,2}\left(m_{Z}\right)$, where $\Delta_{A}, \Delta_{\mu}^{(1,2)}$ are loop corrections [32, 35-38]. The exact forms of the radiative corrections to $\mu$ and $M_{A}$ are not needed for the discussion here, though we do note that the dominant contribution to $\Delta_{\mu}^{(1)}$ at large $\tan \beta$ contains a term which is proportional to $h_{t}^{2} \tan ^{2} \beta$, whereas the dominant contribution to $M_{A}^{2}$ contains terms proportional to $h_{t}^{2} \tan \beta$ and $h_{b}^{2} \tan \beta$. The radiative corrections between the values of the quantities $\mu^{2}, m_{1,2}^{2}$ at $Q$ and the electroweak scale are well known.

It is clear from (2.3) and (2.4) that departures of the input supersymmetry-breaking contributions to $m_{1,2}^{2}$ from their universal values in the CMSSM, as permitted in the NUHM, induce corresponding changes in the allowed values of $\mu$ and $M_{A}^{2}$, respectively. We evaluate all the relevant radiative corrections in our analysis: the sensitivity of $M_{A}$ to $m_{t}$ and $m_{b}$ was discussed extensively in [17], and we do not discuss the issue any further here.

\footnotetext{
${ }^{1}$ See [20], where the full one-loop corrections have been calculated.
} 
Spin-independent elastic $\chi$-nucleon scattering is controlled by the following effective four-fermion Lagrangian:

$$
\mathcal{L}=\alpha_{3 i} \bar{\chi} \chi \overline{q_{i}} q_{i}
$$

which is to be summed over the quark flavours $q$, and the subscript $i$ labels up-type quarks $(i=1)$ and down-type quarks $(i=2)$. The model-dependent coefficients $\alpha_{3 i}$ include terms $\propto 1 / m_{H_{1,2}}^{2}$ [39], where $H_{1,2}$ are the two scalar Higgs bosons in the MSSM, in which it is well known that the lighter one, $H_{2}$, must have a mass $\sim 120 \mathrm{GeV}$, whereas the heavier one, $H_{1}$, has a mass very similar to $M_{A}$. Hence the elastic cold dark matter scattering cross section also increases for small $M_{A}$ [5]. The magnitude of the cross section depends on hadronic matrix elements related to the $\pi$-nucleon $\Sigma$ term, for which values between $\sim 64$ and $\sim 45 \mathrm{MeV}$ are frequently quoted. In the estimates of the $\chi$-nucleon scattering used here, we assume $\Sigma=64 \mathrm{MeV}$, which yields relatively large cross sections. Smaller regions of the NUHM parameter space would be excluded if we used a smaller estimate of $\Sigma$ [19], so this assumption maximizes the possible impact of the CDMS constraint.

\section{Analysis of NUHM parameter planes}

In order to exemplify the possible effects of the $B_{s} \rightarrow \mu^{+} \mu^{-}$and CDMS constraints, we display some specific NUHM $\left(\mu, m_{A}\right)$ planes for different values of $\tan \beta, m_{1 / 2}$ and $m_{0}$, exhibiting the interplay of the different experimental, phenomenological and theoretical constraints. We first consider the case $\tan \beta=10, m_{1 / 2}=300 \mathrm{GeV}$ and $m_{0}=100 \mathrm{GeV}$, shown in panel (a) of figure 1. This value of $\tan \beta$ is towards the lower end of the range where we find generic CMSSM models satisfying all the constraints, for both signs of $\mu$, except for the $g_{\mu}-2$ constraint that is satisfied only for positive $\mu$. The most important constraints for $\mu>0$ are those due to the chargino mass, shown as a vertical black dashed line at low $\mu \sim 100 \mathrm{GeV}$, and the GUT stability constraint at larger $\mu \sim 650 \mathrm{GeV}$, shown as a near-vertical black dash-dotted line that turns horizontal at low $M_{A} \sim 250 \mathrm{GeV}$. Regions between these lines are consistent with both constraints, but we also note a small excluded (brick-red) 'sugarloaf' around $\mu \sim 300 \mathrm{GeV}$ that extends up to $M_{A} \sim 300 \mathrm{GeV}$, where the LSP would have been the lighter stau, $\tilde{\tau}_{1}$. For $\mu<0$, a large region is excluded by $b \rightarrow s \gamma$ decay, as shown by the green (medium) shading here and in subsequent figures. The excluded regions where the $\tilde{\nu}_{e}$ would be the LSP (or become tachyonic) are shaded (dark) blue: in this panel, they lie beyond the GUT stability region. The red dash-dotted line marks the LEP Higgs constraint ${ }^{2}$, which in this case excludes the lower part of the region allowed by GUT stability, and requires $M_{A} \gtrsim 460 \mathrm{GeV}$. Finally, in this panel, regions with $\mu>0$ are allowed by $g_{\mu}-2^{3}$, whereas regions with $\mu<0$ are disallowed at the $2-\sigma$ level.

Within the region allowed by the other constraints, we note that there is a near-vertical WMAP strip extending upwards. All of this WMAP strip is allowed comfortably by the current $B_{s} \rightarrow \mu^{+} \mu^{-}$constraint, since the branching ratio for $B_{s} \rightarrow \mu^{+} \mu^{-}$is very close to its Standard Model value $\sim 3.9 \times 10^{-9}$. It is dubious whether even the LHC would be

\footnotetext{
${ }^{2}$ This is evaluated following the likelihood approach described in 40].

${ }^{3}$ We assume $\delta a_{\mu}$, where $a_{\mu} \equiv\left(g_{\mu}-2\right) / 2$, to be within the range 6.8 to $43.6 \times 10^{-10}$ at the $2-\sigma$ level.
} 

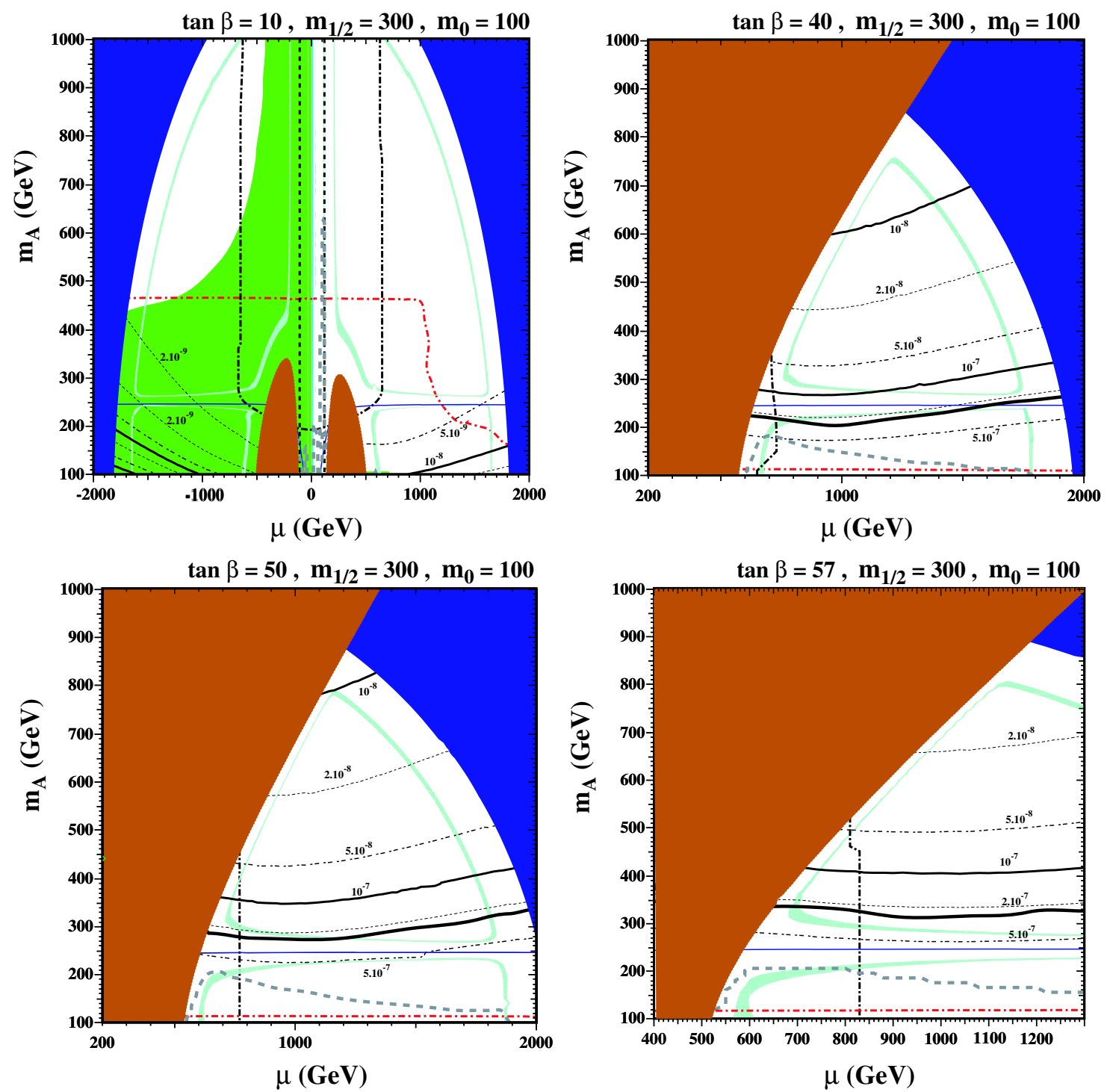

Figure 1: Allowed regions in the $\left(\mu, M_{A}\right)$ planes for $m_{1 / 2}=300 \mathrm{GeV}$ and $m_{0}=100 \mathrm{GeV}$, for (a) $\tan \beta=10$, (b) $\tan \beta=40$, (c) $\tan \beta=50$ and (d) $\tan \beta=57$. In each panel, the near-horizontal solid blue line is the contour where $M_{A}=2 m_{\chi}$, and the turquoise strips are those where the relic neutralino LSP density falls within the range favoured by WMAP and other cosmological and astrophysical observations. The LEP chargino limit is shown as a dashed black line and the GUT stability constraint as a dot-dashed black line. The regions disallowed by $b \rightarrow s \gamma$ are shaded green, and those disallowed because the $\tilde{\tau}_{1}$ or the $\tilde{\nu}_{e}$ would be the LSP are shaded brick-red and dark blue, respectively. The Higgs constraint is the largely horizonal red dot-dashed line. Contours of the $B_{s} \rightarrow \mu^{+} \mu^{-}$branching ratio are labelled correspondingly, with the current Tevatron limit the boldest black line, and the CDMS constraint is shown as a thick dashed grey line. In panel a), the $g_{\mu}-2$ constraint is satisfied when $\mu>0$. In the remaining three panels, the contribution to $g_{\mu}-2$ is too large at the $2-\sigma$ level.

able to distinguish the NUHM from the Standard Model for these values of $\tan \beta, m_{1 / 2}$ and $m_{0}$. Other regions with acceptable relic densities either have a small Higgs mass or 
violate the GUT constraint. These include the lower strip which extends upwards from $M_{A}=100 \mathrm{GeV}$ and turns towards the horizontal at lower $M_{A}$, where rapid $\chi \chi \rightarrow A, H$ annihilation becomes important when $M_{A} \sim 2 m_{\chi}$ (near-horizontal solid blue line) and the WMAP strip in the upper left which is determined by neutralino-sneutrino co-annihilations. Also shown as a dashed grey line is the constraint imposed by the CDMS upper limit on spin-independent elastic cold dark matter scattering. Here and in the subsequent figures, in regions of the NUHM parameter space where the calculated relic LSP density $\Omega_{\chi}$ falls below the WMAP range, the cross section is rescaled by the factor $\Omega_{\chi} / \Omega_{C D M}$, in order to compensate for the the fact that neutralinos could provide only this fraction of the galactic halo. As a result, in this case, not only does the CDMS limit not exclude any of the WMAP strip, it also does not exclude models surviving the LEP chargino constraint.

When $\tan \beta=40,{ }^{4}$ the stau constraint plays a much more important role, as seen in panel (b). It excludes all the parameter space above and to the left of a diagonal line that meets the GUT stability constraint, which is essentially unchanged, at $\mu \sim 700 \mathrm{GeV}$ and $M_{A} \sim 350 \mathrm{GeV}$. The $\tilde{\nu}_{e}$ constraint appears at much larger $\mu$ and $M_{A}$. In this and subsequent panels of this figure, the LEP Higgs constraint excludes only a narrow strip below $M_{A} \sim 120 \mathrm{GeV}$, whereas $(g-2)_{\mu}$ is incompatible with the measurement at the $2-\sigma$ level. The only region allowed by the GUT stability constraint and by WMAP is then a short strip with $\mu \sim 700 \mathrm{GeV}$, which lies above the Higgs constraint and below the solid blue line where $M_{A}=2 m_{\chi}$. All of this strip is excluded by the current $B_{s} \rightarrow \mu^{+} \mu^{-}$constraint (represented by bold solid line). We see also that the CDMS constraint excludes a region of the WMAP strip that is, however, already excluded by the stronger $B_{s} \rightarrow \mu^{+} \mu^{-}$constraint in this case. As already mentioned, the CDMS limit is rescaled when the calculated $\Omega_{\chi}$ falls below the WMAP range, which is responsible for the droop in the dashed grey line for $\mu \sim 600 \mathrm{GeV}$. In this and subsequent figures, the CDMS limit is also shown in regions where $\Omega_{\chi}$ exceeds the WMAP range, even though this region is disallowed by cosmology.

In panel (c) for $\tan \beta=50$, the stau LSP constraint meets the GUT stability constraint at a somewhat higher values of $M_{A} \sim 450 \mathrm{GeV}$. The region by WMAP and GUT stability is now bisected by the $M_{A}=2 m_{\chi}$ line, with strips both above and below this line. The lower strip, where $m_{\chi}>M_{A} / 2$, is already excluded by the present $B_{s} \rightarrow \mu^{+} \mu^{-}$constraint, and the upper strip lies only just beyond the present sensitivity. The lower WMAP strip would also be almost excluded by the CDMS constraint. Finally, in panel (d) for $\tan \beta=57$, which is close to the maximum value for which we find generic solutions to the electroweak vacuum conditions, we see that the stau LSP constraint now intersects the GUT stability constraint at $M_{A} \sim 550 \mathrm{GeV}$, with the $\tilde{\nu}_{e}$ LSP constraint appearing only at larger values of $\mu$. There are again two strips allowed by WMAP, above and below the line where $M_{A}=2 m_{\chi}$. However, in this case, as well as the lower WMAP strip, a large portion of the upper strip is also already excluded by $B_{s} \rightarrow \mu^{+} \mu^{-}$, reflecting the greater power of this constraint as $\tan \beta$ increases. In this case, the CDMS constraint almost excludes the lower WMAP strip.

\footnotetext{
${ }^{4}$ For this and higher values of $\tan \beta$, we find consistent electroweak vacua mostly only for positive values of $\mu$.
} 

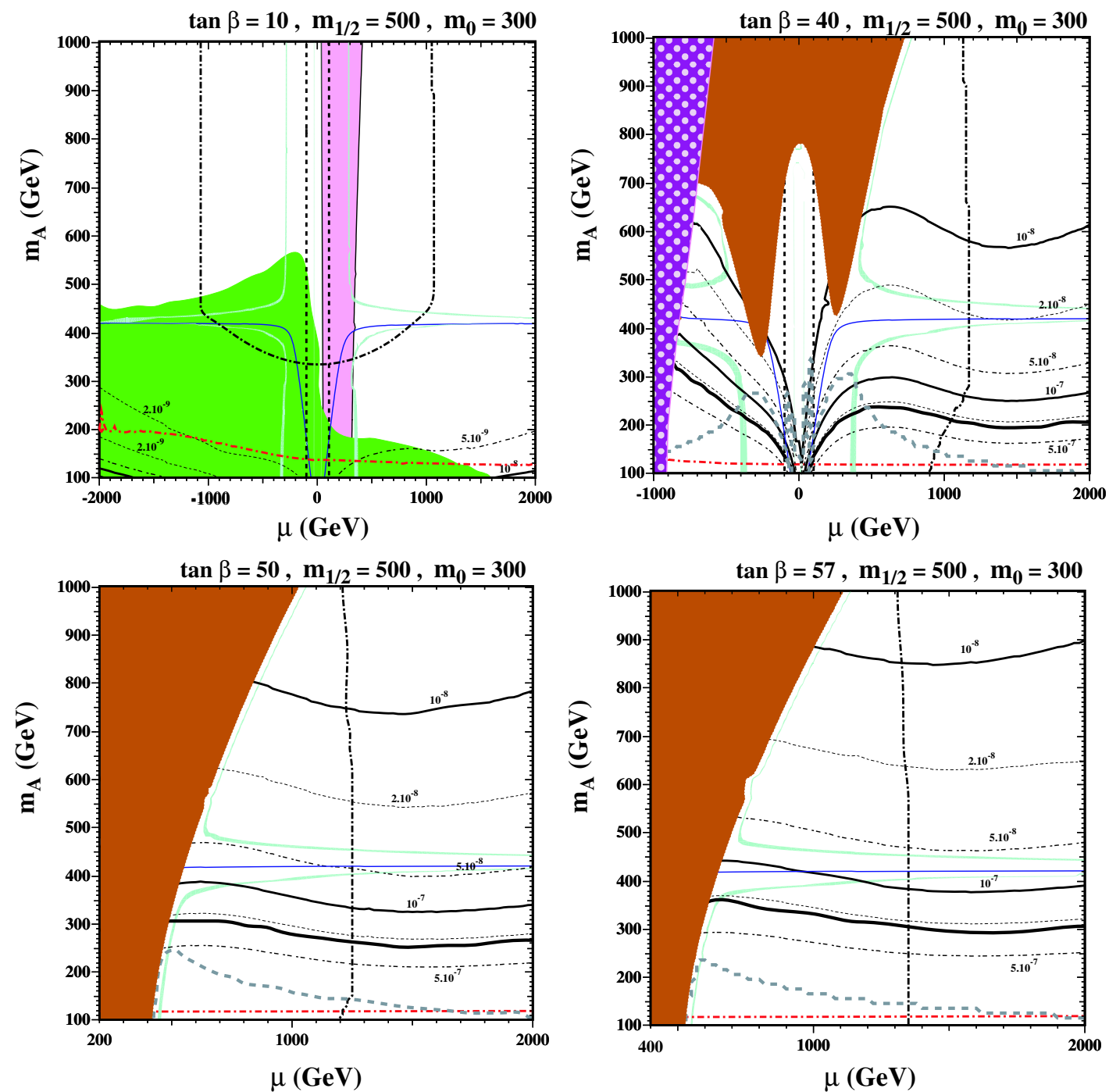

Figure 2: As in figure 1, for the same values of $\tan \beta$ but for the choices $m_{1 / 2}=500 \mathrm{GeV}$ and $m_{0}=300 \mathrm{GeV}$. There is no electroweak symmetry breaking in the polka-dotted region of panel (b). In panel a), the vertical pink shaded region shows the region of $g_{\mu}-2$ allowed at the 2- $\sigma$ level. In the remaining three panels, $g_{\mu}-2$ is allowed when $\mu>0$.

Some qualitatively similar features are seen in figure 2, which displays analogous panels for the case $m_{1 / 2}=500 \mathrm{GeV}, m_{0}=300 \mathrm{GeV}$. For $\tan \beta=10$, both signs of $\mu$ are equally possible, whereas we find no consistent electroweak vacuum in the polka-dotted region for $\tan \beta=40$, and no solutions with $\mu<0$ for the larger values of $\tan \beta$. In all the panels, the GUT stability constraint provides the right boundary of the allowed region at $\mu \sim 1100$ to $1350 \mathrm{GeV}$ and, in panel (a) also a lower limit on $M_{A} \sim 350 \mathrm{GeV}$. For $\mu>0$, the LEP constraint on the chargino mass again supplies the left boundary in panel (a) for $\tan \beta=10,{ }^{5}$ and partially in panel (b) for $\tan \beta=40$. The rest of the left boundary for $\mu>0$ in panel (b), and the entire left boundaries in panels (c, d) for $\tan \beta=50,57$,

\footnotetext{
${ }^{5}$ In panel (a), regions outside the near-vertical (pink) band at $\mu \sim 200 \mathrm{GeV}$ are excluded by $g_{\mu}-2$. In
} 
respectively, are provided by the stau LSP constraint. The bottom boundaries of the allowed regions in panels (b, c, d) are provided by the LEP Higgs constraint.

As in panels $(\mathrm{c}, \mathrm{d})$ of figure 1, each panel features a pair of WMAP strips, above and below the $M_{A}=2 m_{\chi}$ line. When $\tan \beta=10$, the entire WMAP strips are allowed by $B_{s} \rightarrow \mu^{+} \mu^{-}$, and sensitivity to the Standard Model prediction would be required to challenge any part of them. However, in the remaining panels, increasing parts of the lower WMAP strips are excluded by $B_{s} \rightarrow \mu^{+} \mu^{-}$as $\tan \beta$ increases. However, in each case sensitivity to $B_{s} \rightarrow \mu^{+} \mu^{-}$below $10^{-8}$ would be required to explore all of the upper WMAP strip. The CDMS constraint lies outside the GUT stability region for $\tan \beta=10$. On the other hand, it excludes a somewhat larger part of the lower WMAP strip than does $B_{s} \rightarrow \mu^{+} \mu^{-}$for $\tan \beta=40$, whereas the $B_{s} \rightarrow \mu^{+} \mu^{-}$constraint is stronger for $\tan \beta=50$ and 57 .

Both the stau LSP and GUT stability constraints weakened between figures 1 and 2. the culprit being the increased value of $m_{0}$, which makes the stau heavier, and also makes the vacuum more likely to be stable. Therefore, in order to display more clearly the interplay of $B_{s} \rightarrow \mu^{+} \mu^{-}$with the line where $M_{A}=2 m_{\chi}$ and the pairs of upper and lower WMAP strips, we now consider a series of cases with relatively large $m_{0}=1000 \mathrm{GeV}$, for which the stau and GUT stability constraints are irrelevant for large ranges of $\mu$ and $M_{A}$. Panels (a, b, c, d) of figure 3 show the cases $m_{1 / 2}=300,500,1000$ and $1500 \mathrm{GeV}$, respectively, all for $\tan \beta=57$. The regions allowed by $(g-2)_{\mu}$ at the $2-\sigma$ level are shown explicitly in panels (b) and (c): at this level, the entire $\mu>0$ region is allowed in panel (a) and disallowed in panel (d). We notice that the lines where $M_{A}=2 m_{\chi}$ move upwards as $m_{1 / 2}$ increases, reflecting the fact that $m_{\chi} \propto m_{1 / 2}$, approximately. On the other hand, the line representing the current upper bound on $B_{s} \rightarrow \mu^{+} \mu^{-}$is relatively insensitive to both $m_{1 / 2}$ and $\mu$. For this reason, whereas $B_{s} \rightarrow \mu^{+} \mu^{-}$already excludes the lower WMAP strip for the choice $m_{1 / 2}=300 \mathrm{GeV}$ in panel (a) [compare also its impacts in panels (d) of figures 1 and 2], $B_{s} \rightarrow \mu^{+} \mu^{-}$is currently sensitive to only progressively smaller fractions of the lower WMAP strip as $m_{1 / 2}$ increases in panels (b, c, d). By comparison, the CDMS constraint excludes only part of the lower WMAP strip in panel (a), but also a portion of the upper WMAP strip at small $\mu$. The peculiar shape of the CDMS curve in panel a) is caused by our scaling to the relic density, which is particularly important when $m_{\chi} \approx M_{A} / 2$. In panels (b) and (c), CDMS excludes slightly more of the lower WMAP strip than does $B_{s} \rightarrow \mu^{+} \mu^{-}$, but this advantage would be removed if one adopted the lower value $\Sigma=45 \mathrm{MeV}$. The CDMS constraint has no significant impact in panel (d).

To summarize our findings on the current impact of $B_{s} \rightarrow \mu^{+} \mu^{-}$in the $\left(\mu, M_{A}\right)$ planes: its importance increases with $\tan \beta$, and for large values it may exclude substantial parts of the WMAP strip where $M_{A}<2 m_{\chi}$. The impact of $B_{s} \rightarrow \mu^{+} \mu^{-}$does not vary rapidly with $m_{0}$, but is relatively less important as $m_{1 / 2}$ increases.

We now turn to some examples of parameter $\left(M_{A}, \tan \beta\right)$ planes, shown in figure 4 , which represent orthogonal projections of the NUHM parameter space that are often

the remaining three panels, all regions shown with $\mu>0$ are allowed by $g_{\mu}-2$. In both panels (a) and (b) there are regions where $\mu<0$ that are allowed by the other constraints, but disallowed by $g_{\mu}-2$. 

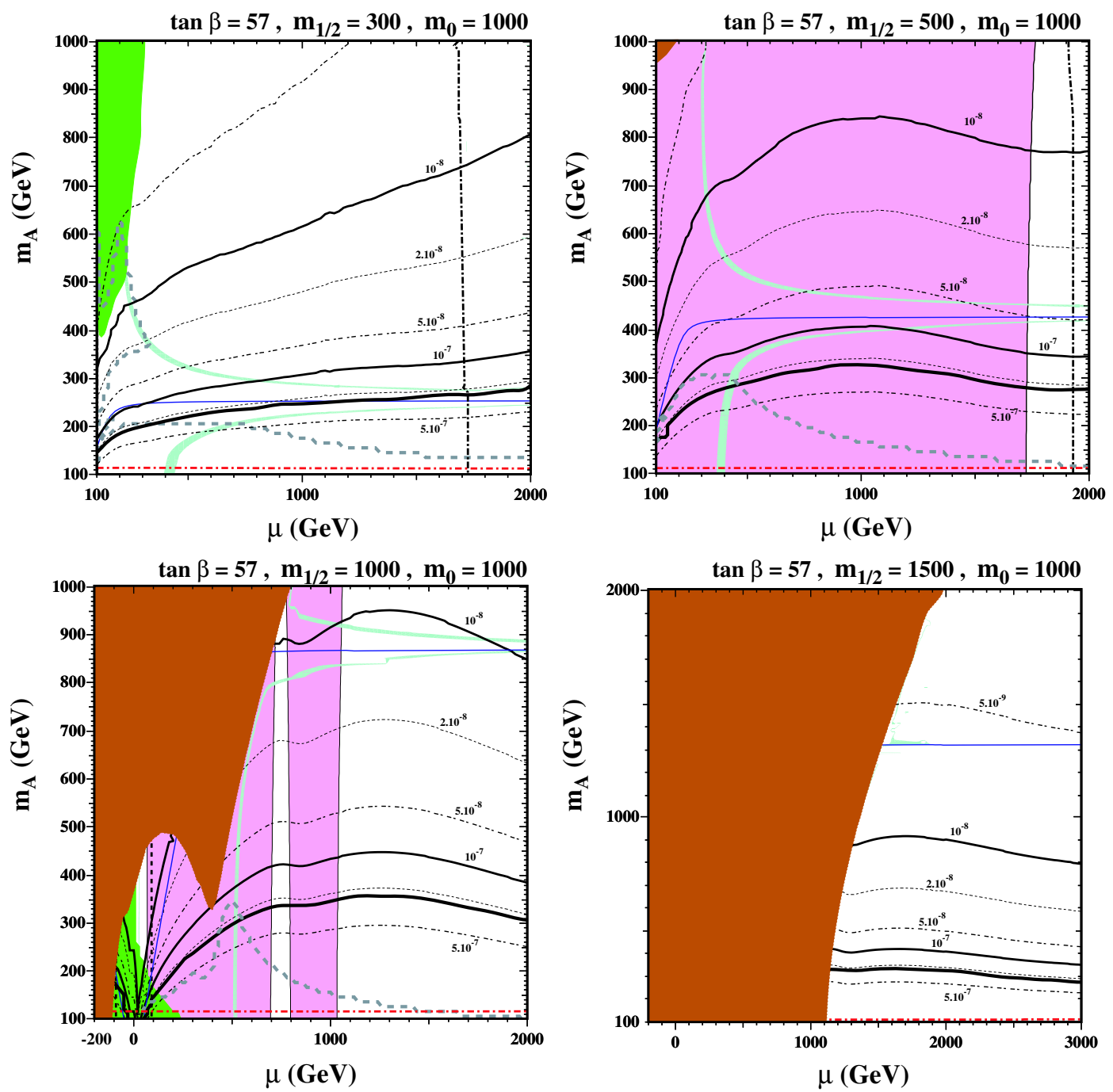

Figure 3: As in figure 1, but for the choices $m_{1 / 2}=$ (a) $300 \mathrm{GeV}$, (b) $500 \mathrm{GeV}$, (c) $1000 \mathrm{GeV}$ and (d) $1500 \mathrm{GeV}$, all for $\tan \beta=57$ and $m_{0}=1000 \mathrm{GeV}$. The regions allowed by the $g_{\mu}-2$ constraint are shaded pink (light grey) in panels (b) and (c). In panel (a), the region with $\mu>0$ is allowed by $g_{\mu}-2$, whereas that in panel (d) is disallowed at the $2-\sigma$ level.

favoured in analyses of MSSM Higgs phenomenology [41]. For convenience of comparison, our examples are taken from [4]. We see in panel (a) for $\mu=200 \mathrm{GeV}, m_{1 / 2}=250 \mathrm{GeV}$ and $m_{0}=1000 \mathrm{GeV}$ that $b \rightarrow s \gamma$ excludes regions at both large and small values of $\left(M_{A}, \tan \beta\right)$. The strip allowed by WMAP is relatively broad, with a portion to the right of the blue line where $M_{A}=2 m_{\chi}$, and another part at low $\tan \beta$, but the latter is excluded by the LEP Higgs constraint. Only the parts of the plane with $\tan \beta \gtrsim 19$ are allowed by $g_{\mu}-2$. In this case, only a small region at small $M_{A}$ and large $\tan \beta$ is excluded by $B_{s} \rightarrow \mu^{+} \mu^{-}$. On the other hand, the CDMS experiment excludes all parts of the WMAP strip that are allowed by $g_{\mu}-2{ }^{6}$

\footnotetext{
${ }^{6}$ This latter conclusion would, however, no longer hold if one adopted $\Sigma=45 \mathrm{MeV}$.
} 

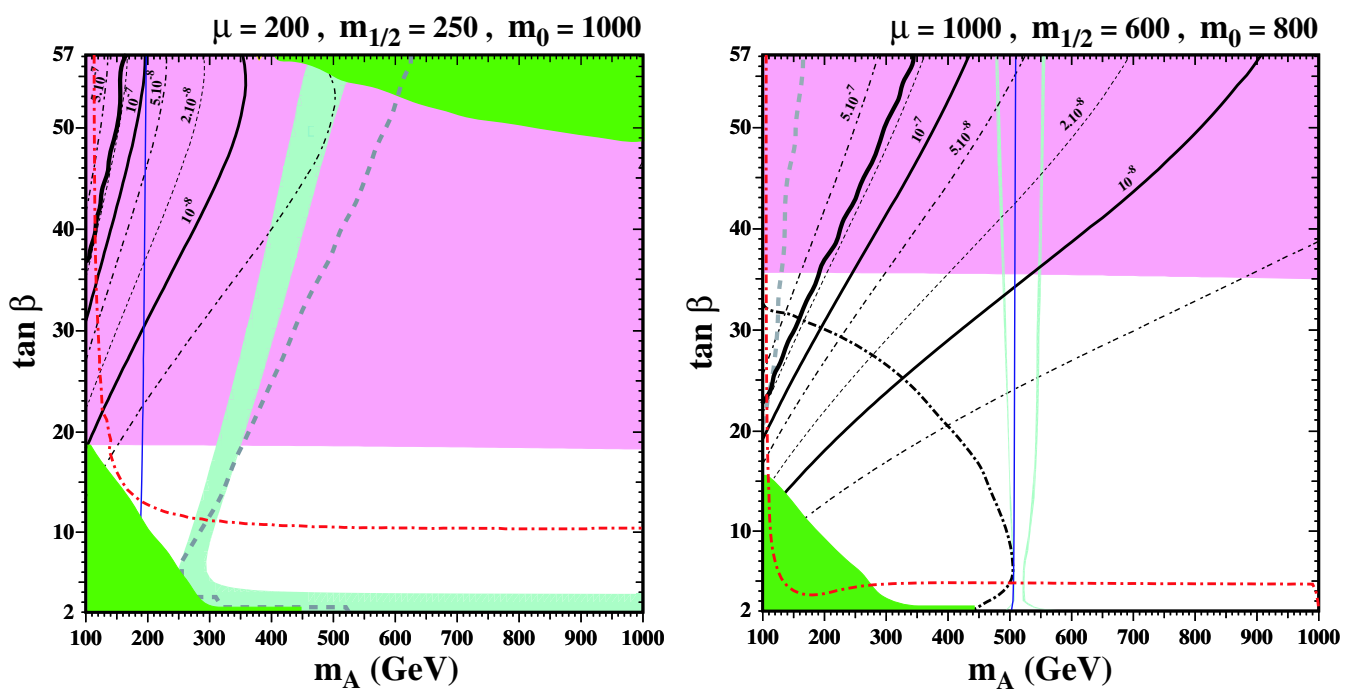

Figure 4: Allowed regions in the $\left(M_{A}, \tan \beta\right)$ planes for (a) $\mu=1000 \mathrm{GeV}, m_{1 / 2}=600 \mathrm{GeV}$ and $m_{0}=800 \mathrm{GeV}$ and (b) $\mu=800 \mathrm{GeV}, m_{1 / 2}=250 \mathrm{GeV}$ and $m_{0}=1000 \mathrm{GeV}$. The constraints are displayed in the same way as in in figure 1 .

Turning now to panel (b) of figure 1 for $\mu=1000 \mathrm{GeV}, m_{1 / 2}=600 \mathrm{GeV}$ and $m_{0}=$ $800 \mathrm{GeV}$, we see that $b \rightarrow s \gamma$ now excludes only a region at small values of $\left(M_{A}, \tan \beta\right)$, and that the requirement of vacuum stability up to the GUT scale also excludes a region at $M_{A} \lesssim 500 \mathrm{GeV}$. The WMAP strip is now much narrower than in panel (a), with portions on either side of the line where $M_{A}=2 m_{\chi}$. The LEP Higgs constraint is weaker, excluding only $\tan \beta \lesssim 5$, whereas the $g_{\mu}-2$ constraint is stronger, excluding the region with $\tan \beta \lesssim 35$. In this case, neither the $B_{s} \rightarrow \mu^{+} \mu^{-}$nor the dark matter scattering constraints have any impact on the allowed portions of the WMAP strips at larger $\tan \beta$.

As already mentioned, $\left(M_{A}, \tan \beta\right)$ planes are often considered in discussions of MSSM Higgs phenomenology. These two examples show that only small parts of these planes may be allowed by the various theoretical, phenomenological and cosmological constraints. In the particular examples studied, $B_{s} \rightarrow \mu^{+} \mu^{-}$is not yet an important constraint, whereas the search for astrophysical dark matter may be. Generic $\left(M_{A}, \tan \beta\right)$ regions may be allowed if one exploits the flexibility of the NUHM to vary $\mu, m_{1 / 2}$ and $m_{0}$ independently at fixed $M_{A}$ and $\tan \beta$. We plan to return to a more detailed discussion of $\left(M_{A}, \tan \beta\right)$ planes in the future.

\section{Summary and prospects}

We have shown in this paper that the current $B_{s} \rightarrow \mu^{+} \mu^{-}$constraint already imposes significant constraints on the NUHM, excluding generic regions with small $M_{A}$ and large $\tan \beta$ that would have been allowed by the other theoretical, phenomenological and cosmological constraints. The direct search for the scattering of cold dark matter also excludes some regions of the NUHM parameter space, but the $B_{s} \rightarrow \mu^{+} \mu^{-}$constraint is stronger in most of the cases we have studied. 
Experiments at the Tevatron and then the LHC are expected to increase greatly the sensitivity to $B_{s} \rightarrow \mu^{+} \mu^{-}$. Our analysis shows that, in many NUHM cases, this improved sensitivity would have good prospects for detecting $B_{s} \rightarrow \mu^{+} \mu^{-}$. For example, sensitivity to $B R\left(B_{s} \rightarrow \mu^{+} \mu^{-}\right) \sim 10^{-8}$ would give access to essentially all the $\left(\mu, M_{A}\right)$ planes for $m_{1 / 2}=300 \mathrm{GeV}, m_{0}=100 \mathrm{GeV}$ and $\tan \beta \gtrsim 40$, as seen in figure 1. On the other hand, in the case of larger $m_{1 / 2}=500 \mathrm{GeV}$ and $m_{0}=300 \mathrm{GeV}$, shown in figure 2, the allowed regions of the WMAP strips extend to larger values of $M_{A}$ that would require greater sensitivity to $B R\left(B_{s} \rightarrow \mu^{+} \mu^{-}\right)$. The same effect is seen even for the largest studied value of $\tan \beta=57$ for several different choices of larger values of $m_{1 / 2}$ and $m_{0}$, as seen in figure 3. Sensitivity to $B R\left(B_{s} \rightarrow \mu^{+} \mu^{-}\right) \sim 10^{-8}$ would not be sufficient to explore any new region of the $\left(M_{A}, \tan \beta\right)$ plane for the choice $\mu=200 \mathrm{GeV}, m_{1 / 2}=250 \mathrm{GeV}$ and $m_{0}=1000 \mathrm{GeV}$, but would explore all the allowed region for $\mu=1000 \mathrm{GeV}, m_{1 / 2}=600 \mathrm{GeV}$ and $m_{0}=800 \mathrm{GeV}$, as seen in panels (a) and (b) of figure 4 , respectively.

We conclude that $B_{s} \rightarrow \mu^{+} \mu^{-}$is already an important constraint on the NUHM parameter space, and that, within this framework, it would have excellent prospects for a future detection of indirect effects of supersymmetry.

\section{Acknowledgments}

The work of K.A.O. and V.C.S. was supported in part by DOE grant DE-FG02-94ER40823. The work of Y.S. was supported in part by the NSERC of Canada.

\section{References}

[1] V. Berezinsky et al., Neutralino dark matter in supersymmetric models with nonuniversal scalar mass terms, Astropart. Phys. 5 (1996) 1 hep-ph/9508249;

P. Nath and R. Arnowitt, Non-universal soft SUSY breaking and dark matter, Phys. Rev. D 56 (1997) 2820 hep-ph/9701301;

A. Bottino, F. Donato, N. Fornengo and S. Scopel, Probing the supersymmetric parameter space by WIMP direct detection, Phys. Rev. D 63 (2001) 125003 hep-ph/0010203.

[2] M. Drees, M.M. Nojiri, D.P. Roy and Y. Yamada, Light higgsino dark matter, Phys. Rev. D 56 (1997) 276 hep-ph/9701219;

see also M. Drees et al., Scrutinizing LSP dark matter at the LHC, Phys. Rev. D 63 (2001) 035008 hep-ph/0007202.

[3] J.R. Ellis, T. Falk, G. Ganis, K.A. Olive and M. Schmitt, Charginos and neutralinos in the light of radiative corrections: sealing the fate of higgsino dark matter, Phys. Rev. D 58 (1998) 095002 hep-ph/9801445;

J.R. Ellis, T. Falk, G. Ganis and K.A. Olive, Supersymmetric dark matter in the light of lep and the Tevatron collider, Phys. Rev. D 62 (2000) 075010 hep-ph/0004169.

[4] J.R. Ellis, K.A. Olive and Y. Santoso, The MSSM parameter space with non-universal Higgs masses, Phys. Lett. B 539 (2002) 107 hep-ph/0204192;

J.R. Ellis, T. Falk, K.A. Olive and Y. Santoso, Exploration of the MSSM with non-universal Higgs masses, Nucl. Phys. B 652 (2003) 259 hep-ph/0210205.

[5] J.R. Ellis, A. Ferstl, K.A. Olive and Y. Santoso, Direct detection of dark matter in the MSSM with non-universal Higgs masses, Phys. Rev. D 67 (2003) 123502 hep-ph/0302032]. 
[6] R. Arnowitt, B. Dutta and Y. Santoso, Coannihilation effects in supergravity and D-brane models, Nucl. Phys. B 606 (2001) 59 hep-ph/0102181;

V. Bertin, E. Nezri and J. Orloff, Neutralino dark matter beyond cmssm universality, JHEP 02 (2003) 046 hep-ph/0210034;

S. Profumo, Neutralino dark matter, B- $\tau$ Yukawa unification and non- universal sfermion masses, Phys. Rev. D 68 (2003) 015006 hep-ph/0304071;

D.G. Cerdeno and C. Muñoz, Neutralino dark matter in supergravity theories with non-universal scalar and gaugino masses, JHEP 10 (2004) 015 hep-ph/0405057;

H. Baer, A. Mustafayev, S. Profumo, A. Belyaev and X. Tata, Direct, indirect and collider detection of neutralino dark matter in SUSY models with non-universal Higgs masses, JHEP 07 (2005) 065 hep-ph/0504001.

[7] LeP Working Group for Higgs boson searches collaboration, R. Barate et al., Search for the standard model Higgs boson at lep, Phys. Lett. B 565 (2003) 61 hep-ex/0306033;

Search for neutral Higgs bosons at LEP, paper submitted to ICHEP04, Beijing, LHWG-NOTE-2004-01, ALEPH-2004-008, DELPHI-2004-042, L3-NOTE-2820, OPAL-TN-744, http://lephiggs.web.cern.ch/LEPHIGGS/papers/August2004_MSSM/index.html.

[8] CLEO collaboration, S. Chen et al., Branching fraction and photon energy spectrum for $b \rightarrow s \gamma$, Phys. Rev. Lett. 87 (2001) 251807 hep-ex/0108032;

Belle collaboration, P. Koppenburg et al., An inclusive measurement of the photon energy spectrum in $b \rightarrow$ s $\gamma$ decays, Phys. Rev. Lett. 93 (2004) 061803 hep-ex/0403004;

BABAR collaboration, B. Aubert et al., Determination of the branching fraction for inclusive decays $B \rightarrow X_{s} \gamma$, hep-ex/0207076.

[9] M. Ciuchini, G. Degrassi, P. Gambino and G.F. Giudice, Next-to-leading QCD corrections to $B \rightarrow X_{s} \gamma$ : standard model and two-Higgs doublet model, Nucl. Phys. B 527 (1998) 21 hep-ph/9710335;

M. Ciuchini, G. Degrassi, P. Gambino and G.F. Giudice, Next-to-leading QCD corrections to $B \rightarrow X_{s} \gamma$ in supersymmetry, Nucl. Phys. B 534 (1998) 3 hep-ph/9806308;

G. Degrassi, P. Gambino and G.F. Giudice, $B \rightarrow X_{s} \gamma$ in supersymmetry: large contributions beyond the leading order, JHEP 12 (2000) 009 hep-ph/0009337;

M. Carena, D. Garcia, U. Nierste and C.E.M. Wagner, $B \rightarrow s \gamma$ and supersymmetry with large $\tan \beta$, Phys. Lett. B 499 (2001) 141 hep-ph/0010003;

P. Gambino and M. Misiak, Quark mass effects in $\bar{B} \rightarrow X_{s} \gamma$, Nucl. Phys. B 611 (2001) 338 hep-ph/0104034;

D.A. Demir and K.A. Olive, $B \rightarrow X_{s} \gamma$ in supersymmetry with explicit CP-violation, Phys. Rev. D 65 (2002) 034007 hep-ph/0107329;

F. Borzumati, C. Greub and Y. Yamada, Beyond leading-order corrections to $\bar{B} \rightarrow X_{s} \gamma$ at large $\tan \beta$ : the charged-Higgs contribution, Phys. Rev. D 69 (2004) 055005

hep-ph/0311151;

T. Hurth, Present status of inclusive rare B decays, Rev. Mod. Phys. $\mathbf{7 5}$ (2003) 1159 hep-ph/0212304.

[10] C.L. Bennett et al., First year Wilkinson microwave anisotropy probe (WMAP) observations: preliminary maps and basic results, Astrophys. J. Suppl. 148 (2003) 1 astro-ph/0302207; WMAP collaboration, D.N. Spergel et al., First year Wilkinson microwave anisotropy probe (WMAP) observations: determination of cosmological parameters, Astrophys. J. Suppl. 148 (2003) 175 astro-ph/0302209. 
[11] Muon G-2 collaboration, G.W. Bennett et al., Measurement of the negative muon anomalous magnetic moment to 0.7-ppm, Phys. Rev. Lett. 92 (2004) 161802 hep-ex/0401008;

M. Davier, S. Eidelman, A. Hocker and Z. Zhang, Updated estimate of the muon magnetic moment using revised results from $e^{+} e^{-}$annihilation, Eur. Phys. J. C 31 (2003) 503 hep-ph/0308213;

K. Hagiwara, A.D. Martin, D. Nomura and T. Teubner, Predictions for g-2 of the muon and alpha $(Q E D)\left(M\left(Z^{2}\right)\right)$, Phys. Rev. D 69 (2004) 093003 hep-ph/0312250;

J.F. de Troconiz and F.J. Yndurain, The hadronic contributions to the anomalous magnetic moment of the muon, Phys. Rev. D 71 (2005) 073008 hep-ph/0402285;

K. Melnikov and A. Vainshtein, Hadronic light-by-light scattering contribution to the muon anomalous magnetic moment revisited, Phys. Rev. D 70 (2004) 113006 hep-ph/0312226;

M. Passera, The standard model prediction of the muon anomalous magnetic moment, J. Phys. G31 (2005) R75-R94 hep-ph/0411168.

[12] A. Dedes, H.K. Dreiner and U. Nierste, Correlation of $B / s \rightarrow \mu^{+} \mu^{-}$and $(g-2)(\mu)$ in minimal supergravity, Phys. Rev. Lett. 87 (2001) 251804 hep-ph/0108037.

[13] R. Arnowitt, B. Dutta, T. Kamon and M. Tanaka, Detection of $b / s \rightarrow \mu^{+} \mu^{-}$at the Tevatron run ii and constraints on the SUSY parameter space, Phys. Lett. B 538 (2002) 121 hep-ph/0203069.

[14] S. Baek, P. Ko and W.Y. Song, Implications on SUSY breaking mediation mechanisms from observing $B / s \rightarrow \mu^{+} \mu^{-}$and the muon $(g-2)$, Phys. Rev. Lett. 89 (2002) 271801 hep-ph/0205259;

C.S. Huang and X.H. Wu, $B / s \rightarrow \mu^{+} \mu^{-}$and $B \rightarrow X_{s} \mu^{+} \mu^{-}$in MSSM, Nucl. Phys. B 657 (2003) 304 hep-ph/0212220;

S. Baek, Y.G. Kim and P. Ko, Neutralino dark matter scattering and $B / s \rightarrow \mu^{+} \mu^{-}$in SUSY models, JHEP 02 (2005) 067 hep-ph/0406033.

[15] H. Baer et al., Updated constraints on the minimal supergravity model, JHEP 07 (2002) 050 hep-ph/0205325.

[16] CDF collaboration, F. Abe et al., Search for the decays $B / D O \rightarrow \mu^{+} \mu^{-}$and $B / S 0 \rightarrow \mu^{+} \mu^{-}$ in p $\bar{p}$ collisions at $\sqrt{s}=1.8$ tev, Phys. Rev. D 57 (1998) 3811;

CDF collaboration, D. Acosta et al., Search for $B / S 0 \rightarrow \mu^{+} \mu^{-}$and $B / D 0 \rightarrow \mu^{+} \mu^{-}$decays in p p collisions at $\sqrt{s}=1.9$ 6-tev, Phys. Rev. Lett. 93 (2004) 032001 hep-ex/0403032;

D0 collaboration, V.M. Abazov et al., A search for the flavor-changing neutral current decay $B / S 0 \rightarrow \mu^{+} \mu^{-}$in p p $\bar{p}$ collisions at $\sqrt{s}=1.96$-tev with the d0 detector, Phys. Rev. Lett. 94 (2005) 071802 hep-ex/0410039;

The D0 Collaboration, D0note, 4733-CONF

http://www-d0.fnal.gov/Run2Physics/WWW/results/prelim/B/B21/B21.pdf;

M. Herndon, The CDF and D0 Collaborations, FERMILAB-CONF-04-391-E, published

Proceedings 32nd International Conference on High-Energy Physics (ICHEP 04), Beijing,

China, August 16-22, 2004;

The CDF Collaboration, CDF note 7670

http://www-cdf.fnal.gov/physics/new/bottom/050407.blessed-bsmumu/.

[17] J.R. Ellis, K.A. Olive and V.C. Spanos, On the interpretation of $B / S \rightarrow \mu^{+} \mu^{-}$in the CMSSM, Phys. Lett. B 624 (2005) 47 hep-ph/0504196.

[18] CDMS collaboration, D.S. Akerib et al., Limits on spin-independent wimp nucleon interactions from the two-tower run of the cryogenic dark matter search, Phys. Rev. Lett. 96 (2006) 011302 astro-ph/0509259. 
[19] J.R. Ellis, K.A. Olive, Y. Santoso and V.C. Spanos, Update on the direct detection of supersymmetric dark matter, Phys. Rev. D 71 (2005) 095007 hep-ph/0502001.

[20] C. Bobeth, T. Ewerth, F. Kruger and J. Urban, Analysis of neutral Higgs-boson contributions to the decays $B \overline{/ S} \rightarrow \ell^{+} \ell^{-}$and $\bar{B} \rightarrow K \ell^{+} \ell^{-}$, Phys. Rev. D 64 (2001) 074014 hep-ph/0104284.

[21] H.E. Logan and U. Nierste, $B / S, D \rightarrow \ell^{+} \ell^{-}$in a two-Higgs-doublet model, Nucl. Phys. B 586 (2000) 39 hep-ph/0004139.

[22] T. Inami and C.S. Lim, Effects of superheavy quarks and leptons in low-energy weak processes $K_{l} \rightarrow \mu \bar{\mu}, K^{+} \rightarrow \pi^{+}$neutrino anti-neutrino and $K 0 \rightarrow \bar{K} 0$, Prog. Theor. Phys. 65 (1981) 297, Erratum, Prog. Theor. Phys. 65 (1981) 1772.

[23] G. Buchalla and A.J. Buras, $Q C D$ corrections to rare $K$ and $B$ decays for arbitrary top quark mass, Nucl. Phys. B 400 (1993) 225;

M. Misiak and J. Urban, QCD corrections to fcnc decays mediated by Z-penguins and $W$-boxes, Phys. Lett. B 451 (1999) 161 hep-ph/9901278.

[24] CDF collaboration, Combination of CDF and D0 results on the top-quark mass, hep-ex/0507091.

[25] S.R. Choudhury and N. Gaur, Dileptonic decay of B/S meson in SUSY models with large $\tan \beta$, Phys. Lett. B 451 (1999) 86 hep-ph/9810307;

C.-S. Huang, W. Liao, Q.-S. Yan and S.-H. Zhu, B/s $\rightarrow \ell^{+} \ell^{-}$in a general 2HDM and MSSM, Phys. Rev. D 63 (2001) 114021 hep-ph/0006250;

P.H. Chankowski and L. Slawianowska, $B 0 / D, S \rightarrow \mu^{-} \mu^{+}$decay in the MSSM, Phys. Rev. D 63 (2001) 054012 hep-ph/0008046.

[26] K.S. Babu and C.F. Kolda, Higgs-mediated $B 0 \rightarrow \mu^{+} \mu^{-}$in minimal supersymmetry, Phys. Rev. Lett. 84 (2000) 228 hep-ph/9909476.

[27] C. Bobeth, A.J. Buras, F. Kruger and J. Urban, $Q C D$ corrections to $\bar{B} \rightarrow X / D, S \nu \bar{\nu}, B / \bar{D}, S$ $\rightarrow \ell^{+} \ell^{-}, K \rightarrow \pi \nu \bar{\nu}$ and $K_{l} \rightarrow \mu^{+} \mu^{-}$in the MSSM, Nucl. Phys. B 630 (2002) 87 hep-ph/0112305.

[28] J.K. Mizukoshi, X. Tata and Y. Wang, Higgs-mediated leptonic decays of $B / S$ and $B / D$ mesons as probes of supersymmetry, Phys. Rev. D 66 (2002) 115003 hep-ph/0208078.

[29] C. Bobeth, T. Ewerth, F. Kruger and J. Urban, Enhancement of $B\left(\bar{B} / D \rightarrow \mu^{+} \mu^{-}\right) / B(\bar{B} / s)$ $\rightarrow \mu^{+} \mu^{-}$) in the MSSM with minimal flavour violation and large tan beta, Phys. Rev. D 66 (2002) 074021 hep-ph/0204225.

[30] R. Hempfling, Yukawa coupling unification with supersymmetric threshold corrections, Phys. Rev. D 49 (1994) 6168;

L.J. Hall, R. Rattazzi and U. Sarid, The top quark mass in supersymmetric SO(10) unification, Phys. Rev. D 50 (1994) 7048 hep-ph/9306309];

M. Carena, M. Olechowski, S. Pokorski and C.E.M. Wagner, Electroweak symmetry breaking and bottom-top Yukawa unification, Nucl. Phys. B 426 (1994) 269 hep-ph/9402253;

R. Rattazzi and U. Sarid, The unified minimal supersymmetric model with large yukawa couplings, Phys. Rev. D 53 (1996) 1553 hep-ph/9505428;

H. Eberl, K. Hidaka, S. Kraml, W. Majerotto and Y. Yamada, Improved SUSY QCD corrections to Higgs boson decays into quarks and squarks, Phys. Rev. D 62 (2000) 055006 hep-ph/9912463. 
[31] M. Carena, D. Garcia, U. Nierste and C.E.M. Wagner, Effective lagrangian for the $\bar{T} B H+$ interaction in the MSSM and charged Higgs phenomenology, Nucl. Phys. B 577 (2000) 88 hep-ph/9912516.

[32] D.M. Pierce, J.A. Bagger, K.T. Matchev and R.-j. Zhang, Precision corrections in the minimal supersymmetric standard model, Nucl. Phys. B 491 (1997) 3 hep-ph/9606211.

[33] G. Isidori and A. Retico, Scalar flavour-changing neutral currents in the large- $\tan \beta$ limit, JHEP 11 (2001) 001 hep-ph/0110121;

G. Isidori and A. Retico, $B / s, d \rightarrow \ell^{+} \ell^{-}$and $K_{l} \rightarrow \ell^{+} \ell^{-}$in SUSY models with non- minimal sources of flavour mixing, JHEP 09 (2002) 063 hep-ph/0208159.

[34] A.J. Buras, P.H. Chankowski, J. Rosiek and L. Slawianowska, $\Delta(M(d, s)), B /(D, s) 0 \rightarrow$ $\mu^{+} \mu^{-}$and $b \rightarrow X /$ s $\gamma$ in supersymmetry at large $\tan \beta$, Nucl. Phys. B 659 (2003) 3 hep-ph/0210145.

[35] V.D. Barger, M.S. Berger and P. Ohmann, The supersymmetric particle spectrum, Phys. Rev. D 49 (1994) 4908 hep-ph/9311269.

[36] W. de Boer, R. Ehret and D.I. Kazakov, Predictions of SUSY masses in the minimal supersymmetric grand unified theory, Z. Physik C 67 (1995) 647 hep-ph/9405342].

[37] M. Carena, J.R. Ellis, A. Pilaftsis and C.E.M. Wagner, Higgs-boson pole masses in the MSSM with explicit CP-violation, Nucl. Phys. B 625 (2002) 345 hep-ph/0111245.

[38] J.R. Ellis, G. Ridolfi and F. Zwirner, Radiative corrections to the masses of supersymmetric Higgs bosons, Phys. Lett. B 257 (1991) 83; On radiative corrections to supersymmetric Higgs boson masses and their implications for LEP searches, Phys. Lett. B 262 (1991) 477;

A. Yamada, Radiative corrections to the Higgs masses in the minimal supersymmetric standard model, Phys. Lett. B 263 (1991) 233;

M. Drees and M.M. Nojiri, One loop corrections to the Higgs sector in minimal supergravity models, Phys. Rev. D 45 (1992) 2482;

P.H. Chankowski, S. Pokorski and J. Rosiek, Charged and neutral supersymmetric Higgs boson masses: complete one loop analysis, Phys. Lett. B 274 (1992) 191; One loop corrections to the supersymmetric Higgs boson couplings and lep phenomenology, Phys. Lett. B 286 (1992) 307;

A. Dabelstein, The one loop renormalization of the MSSM Higgs sector and its application to the neutral scalar Higgs masses, Z. Physik C 67 (1995) 495 hep-ph/9409375;

M. Carena, J.R. Ellis, A. Pilaftsis and C.E.M. Wagner, Renormalization-group-improved effective potential for the MSSM Higgs sector with explicit CP-violation, Nucl. Phys. B 586 (2000) 92 hep-ph/0003180;

A. Katsikatsou, A.B. Lahanas, D.V. Nanopoulos and V.C. Spanos, On the radiative corrections to the pseudo-scalar Higgs boson mass, Phys. Lett. B 501 (2001) 69 hep-ph/0011370.

[39] J.R. Ellis, A. Ferstl and K.A. Olive, Re-evaluation of the elastic scattering of supersymmetric dark matter, Phys. Lett. B 481 (2000) 304 hep-ph/0001005; Exploration of elastic scattering rates for supersymmetric dark matter, Phys. Rev. D 63 (2001) 065016 hep-ph/0007113; Constraints from accelerator experiments on the elastic scattering of cmssm dark matter, Phys. Lett. B 532 (2002) 318 hep-ph/0111064.

[40] J.R. Ellis, K.A. Olive, Y. Santoso and V.C. Spanos, Likelihood analysis of the CMSSM parameter space, Phys. Rev. D 69 (2004) 095004 hep-ph/0310356; 
J.R. Ellis, S. Heinemeyer, K.A. Olive and G. Weiglein, Phenomenological indications of the scale of supersymmetry, hep-ph/0602220.

[41] Z. Kunszt and F. Zwirner, Testing the Higgs sector of the minimal supersymmetric standard model at large hadron colliders, Nucl. Phys. B 385 (1992) 3 hep-ph/9203223;

Higgs Working Group collaboration, M. Carena et al., Report of the Tevatron Higgs working group, hep-ph/0010338;

M. Carena, S. Heinemeyer, C.E.M. Wagner and G. Weiglein, Suggestions for benchmark scenarios for MSSM Higgs boson searches at hadron colliders, Eur. Phys. J. C 26 (2003) 601 hep-ph/0202167]; MSSM Higgs boson searches at the Tevatron and the LHC: impact of different benchmark scenarios, Eur. Phys. J. C 45 (2006) 797 hep-ph/0511023;

ALEPH collaboration, Search for neutral MSSM Higgs bosons at LEP, hep-ex/0602042. 